\title{
Responding to incongruous questions: Effects of making reference to pictures on items referring to related versus unrelated terms
}

\author{
GERALD A. WINER, DAVID A. SMITH, and JOYCE HEMPHILL \\ Ohio State University, Columbus, Ohio
}

\begin{abstract}
Adults received incongruous questions in which the relation implied by the question was incorrect, as in "Why is a cat a dog?" Results showed that when the referents in the question were members of the same category, subjects tended to reject the question fewer times and attempt more answers than when the terms were unrelated. There were also more rejections and fewer attempts to answer the questions when a picture was substituted for one of the verbal referents. The results were interpreted in terms of linguistic pragmatics.
\end{abstract}

In a series of studies examining the extent to which children and adults can recognize and overcome the impact of leading questions (Kwock, 1984; Kwock \& Winer, 1986; Winer, Hemphill, \& Craig, in press; Winer, Rasnake, \& Smith, 1987), we presented adults with flagrantly incongruous questions of the form "Why is this an A?" in reference to a picture of an object $B$ that was different from A (e.g., "Why is this a house?" in reference to a picture of a car). We found that children and adults often detected the misleading nature of the question and rejected the question, answering "It isn't an A, it's a B." In some instances, the presence of these obviously misleading questions sensitized subjects to the misleading nature of other types of questions (Kwock \& Winer, 1986; Winer et al., 1987). However, in a recent dissertation examining responses of children to incongruous questions, Smith (1985) reported few rejections of the questions and many attempts to answer clearly unanswerable items. One of several differences between Smith's procedure and those of other studies is that Smith employed only verbal items; that is, his items made no reference to pictures. One goal of the present study was to compare responses to two types of questions, one making reference to pictures, the other presented in a completely verbal manner, in an adult population.

We also varied the extent to which the items mentioned in each question could be related to one another. For some subjects, the referents in each question were members of the same class (e.g., "Why is a cat a dog?" where both referents, cat and dog, are members of the class animals). For other subjects, the referents had no obvious relation. On the basis of linguistic pragmatics (Grice, 1975, 1978), an approach that stresses the role of context in the interpretation of utterances, we hypothesized that the subjects would more frequently attempt to answer the questions

Address correspondence to Gerald A. Winer, Department of Psychology, Ohio State University, Townshend Hall, 1885 Neil Avenue Mall, Columbus, OH 43210. involving terms that were related to one another and would reject fewer of these items than they would unrelated items. That is, the fact that the referents bear a relation to one another could allow the subject to see a connection and interpret the question as having meaning (e.g., in the question "Why is a cat like a dog?").

\section{METHOD}

The sample consisted of 76 college students attending an orientation class. During class each subject received a series of four questions that asked why an object was something else. The questions appeared on a sheet of paper. For approximately half of the sample, the questions involved pictures (i.e., the question "Why is this an A?" was presented with a picture of $B$, which was something other than A). For the remaining subjects, we substituted the label for the picture and asked "Why is a B and A?" For approximately one half of the questions representing each medium, pictorial or verbal, the referents (As and Bs) were members of a common superordinate classs. For the remaining half, the referents bore no such relation to one another. In sum, we employed a $2 \times 2$ (medium $\times$ type of relation) design.

\section{RESULTS AND DISCUSSION}

We scored each question in two ways. First, we determined the number of times a subject attempted to generate a meaningful answer (e.g., "Because they are both animals"). Results of a $2 \times 2 \times 2$ (medium $\times$ type of relation $\times$ gender) ANOVA supported the prediction. There were significantly more attempts to answer the questions containing terms that were related to one another than those containing nonrelated terms $[F(1,68)=35.7$, $p<.001 ; M s=2.9$ and 1.1 for related and nonrelated items, respectively]. Moreover, there were significantly more attempts to answer the items presented verbally than those presented with pictures $[F(1,68)=4.88, p<.04$; $M s=2.39$ and 1.78 for verbal and pictorial items, respectively]. We then scored the data by examining the number of times a subject "rejected" the underlying assumption of the questions (viz., that there was a relation between the referents) by indicating its nonacceptability 
(e.g., by stating "It's not an A, it's a B"' or 'It's a B," or by claiming that the question was ridiculous). The results of a $2 \times 2 \times 2$ (medium $\times$ type of relation $\times$ gender) ANOVA indicated a greater number of rejections when the referent terms were unrelated than when they were related $[F(1,68)=5.14, p<.03$; mean number of rejections $=1.0$ and 1.9 for related and unrelated terms, respectively]. There were also significantly fewer rejections $[F(1,68)=4.18, p<.05]$ among the purely verbal items $(M=1.03)$ than among the pictorial ones $(M=1.80)$.

In certain respects, the effects due to the relation between referents were not surprising. It is, after all, intrinsically difficult to provide answers to questions about the relation of two objects that bear no relation to each other. However, when the referents were related, subjects might have interpreted the question to be asking about the similarity of the referents (e.g., "Why is a B like an A?'). This is precisely what would be expected on the bases of pragmatic accounts of language (e.g, Grice, 1975). The outcome due to the presence or absence of the pictorial referent, although predicted, was still surprising. It, too, might be explained by linguistic pragmatics (Grice, 1975, 1978). That is, a question of the form "Why is a B an A?" includes the terms A and B as well as the verb linking them. In other words, the form of the question directly implies that a B is an A, as would occur if the question were transformed into a direct statement. The subject, who was influenced by the force or the implication of the question, thus tended to see $\mathrm{a} B$ as an $\mathrm{A}$. The alternative form of the question lacks a direct verbal equation of two referent terms; the word this and a picture are substituted for one of the verbal terms. The fact that the question does not as directly imply that a B is an A should thus weaken the effect of the implication, allowing the subject a greater opportunity to reject it. There is an alternative explanation for the verbal-picture effect. It is possible that the purely verbal items might have allowed the subject more freedom to associate and find connections between the referent terms, whereas the concreteness of the pictures might have limited the subject's ability to imagine associations between unrelated items.
In conclusion, the results generally support the position of linguistic pragmatics. Of particular interest in this connection is the possibility suggested by the results that substituting pictures for their equivalent words can alter the implication of a message. The results might also be of some practical significance in that they show some limitations in the extent to which people might be misled by various statements. Moreover, the fact that there was an effect due to classificatory relations between the terms suggests that a paradigm based upon the design of this study might be useful as a tool for determining the extent to which children perceive meaningful relations between terms. For example, one might use the design of this study with young children. Any difference in the number of times they reject questions that could be attributed to the relation between categorical referents, might be taken as evidence that the children are perceiving a categorical relation among the stimuli.

\section{REFERENCES}

Grice, H. (1975). Logic and conversation. In P. Cole \& J. L. Morgan (Eds.), Syntax and semantics: Volume 3. Speech Acts. New York: Academic Press.

GRICE, H. P. (1978). Further notes on logic and conversation. In P. Cole (Ed.), Syntax and semantics: Volume 9. Pragmatics. New York: Academic Press.

Kwock, M. S. (1984). The effect of linguistic and psychosocial factors on children's logical performance. Unpublished doctoral dissertation, Ohio State University, Columbus.

Kwock, M. S., \& WINER, G. A. (1986). Overcoming leading questions: Effects of psychosocial task variables. Journal of Educational Psychology, 78, 289-293.

SMITH, D. A. (1985). An analysis of responses to ambiguous questions. Unpublished doctoral dissertation, Ohio State University, Columbus.

Winer, G. A., Craig, R. K., \& Hemphill, J. (in press). The effect of misleading questions in promoting non-conversation responses in children and adults. Developmental Psychology.

Winer, G. A., RasNaKe, K., \& Smith, D. A. (1987). Language vs. logic: Responses to ambiguous classificatory questions. Journal of Psycholinguistic Research, 16, 311-327.

(Manuscript received for publication April 2, 1987.) 